\title{
Serum Levels of Neutrophil Gelatinase Associated Lipocalin (NGAL) Predicts Hemodialysis After Coronary Angiography in High Risk Patients with Acute Coronary Syndrome.
}

Luis Felipe Reyes ( $\square$ luis.reyes5@unisabana.edu.co)

Universidad de La Sabana https://orcid.org/0000-0003-1172-6539

Diego Fernando Severiche-Bueno

Universidad de La Sabana

Carlos A. Bustamante

Universidad de La Sabana

Sixta Murillo

Universidad de La Sabana

Nilam J. Soni

University of Texas Health Science Center at San Antonio

Marcela Poveda

Fundacion Clinica Abood Shaio

Efrain Gomez

Universidad de La Sabana

Ricardo Buitrago

Universidad de La Sabana

Alejandro Rodriguez

Hospital Universitari de Tarragona Joan XXIII

Research article

Keywords: Contrast-induced Nephropathy, hemodialysis, biomarkers.

Posted Date: March 27th, 2020

DOI: https://doi.org/10.21203/rs.2.17874/v3

License: (c) (1) This work is licensed under a Creative Commons Attribution 4.0 International License.

Read Full License 
Version of Record: A version of this preprint was published at BMC Nephrology on April 22nd, 2020. See the published version at https://doi.org/10.1186/s12882-020-01799-5. 


\section{Abstract}

Background: Contrast-induced nephropathy ( $\mathrm{CIN})$ following a percutaneous coronary intervention (PCI) is the third most common cause of acute kidney injury (AKI) worldwide. Patients who require hemodialysis secondary to CIN have an elevated mortality rate as high as $55 \%$. The current definition of CIN is based on an elevation of creatinine and decrease in urinary output. Creatinine typically increases 48 hours after the contrast exposure, which delays the diagnosis and treatment of CIN. The neutrophil gelatinase associated lipocalin (NGAL) has emerged as a sensitive and specific biomarker of renal injury. Limited data exists about the effectiveness of NGAL to predict CIN in high-risk patients with acute coronary syndrome (ACS) that underwent $\mathrm{PCl}$. The primary aim of this study was to determine the association of serum NGAL levels and the need for hemodialysis after PCI. Methods: This is a prospective, observational study. NGAL levels were measured using ELISA. Blood samples were obtained within the first 6 hours of hospital admission, and 12 and 24 hours after contrast exposure from angiography. The primary outcome was the requirement of hemodialysis. The non-parametric Mann-Whitney $\mathrm{U}$ test was used to test for differences in median serum levels of NGAL. A receiver operating characteristic (ROC) curve was developed to assess the accuracy of NGAL to predict the need for hemodialysis after PCI. Results: A total of 2,875 were screened; however, 45 patients with ACS that underwent $\mathrm{PCl}$ were included. All patients were at high risk of developing CIN defined by Mehran score $>11$ points. The median (IQR) serum concentration of NGAL was significantly higher in patients that required versus did not require hemodialysis (340 [83-384] vs. 169 [100-210], p=0.01). Elevated serum levels of NGAL with a cut-off at 6 hours post $\mathrm{PCl}$ of $281 \mathrm{mg} / \mathrm{dL}$ predicted the need for hemodialysis with an area under the curve of 0.86 (95\% Cl: 0.66-1.00). Conclusions: In patients with ACS undergoing PCl; and high risk of developing $\mathrm{CIN}$, an elevated serum level of NGAL 6 hours after contrast exposure predicts the development of acute kidney injury requiring hemodialysis.

\section{Background}

The incidence of contrast-induced nephropathy (CIN) is low in patients without risk factors $(<5 \%)$ [1], but increases among patients with chronic kidney disease (CKD) [2], particularly in patients with diabetes mellitus (DM), congestive heart failure (CHF), and advanced age [3]. Additionally, the risk of developing $\mathrm{CIN}$ is even higher among patients with acute coronary syndrome (ACS) who undergo percutaneous coronary intervention $(\mathrm{PCl})[4]$. Incidence of $\mathrm{CIN}$ after a $\mathrm{PCl}$ is $<3 \%$ in patients without renal dysfunction but can rise to $40 \%$ in patients with chronic kidney disease besides being associated with higher inhospital mortality $[5,6]$.

$\mathrm{CIN}$ is defined as an acute decrease in renal function after the administration of intravenous contrast without an alternative cause [7]. The most frequently used definition of $\mathrm{CIN}$ is based on the elevation of serum creatinine (SCr) [8]. It is important to note that SCr does not begin to rise until $50 \%$ or more of the kidney glomeruli have been affected [9]. Serum creatinine is neither sensitive nor specific because several conditions can alter its serum concentration $[10,11]$. 
Several scoring systems have been devised to identify patients at high risk of developing CIN after a cardiac intervention [12-16]. Mehran et al created a scoring system that incorporates 8 clinical and procedural variables (hypotension, congestive heart failure, serum creatinine, DM, age $>75$ years, anemia, the volume of contrast, and use of an intra-aortic balloon pump) that have been widely used and validated in different cohorts [17-20].

Given the limitations of SCr to detect patients with subclinical CIN, isolation of a biomarker for acute kidney injury after a PCl would be clinically useful. Neutrophil gelatinase associated-lipocalin (NGAL), also known as lipocalin-2 (LCN2), is a small (25-kDa) glycoprotein covalently stored in granules of mature neutrophils [21]. It is normally expressed at very low levels in a variety of human tissues, including bone marrow, uterus, prostate, salivary gland, stomach, colon, trachea, lung, liver, and kidney [22, 23]. It is rapidly released in distal tubular cells in response to inflammation or injury of nephrons [24]. It can be easily detected in the blood and urine soon after acute kidney injury $[25,26]$. NGAL is emerging as a promising renal biomarker to detect patients with acute kidney injury [27]. Recent studies have shown that NGAL can be used as a diagnostic tool to detect $\mathrm{CIN}$ in patients that undergo an elective PCI with similar performance between urinary and serum NGAL. However, most of the studies in this group of patients used serum NGAL[25, 28-30].

However, limited data are available on the utility of NGAL to predict CIN and the need for hemodialysis in high-risk patients with ACS and this study will attempt to solve this gap in the literature. Therefore, we hypothesized that serum levels of NGAL could predict the development of CIN requiring hemodialysis after $\mathrm{PCl}$ in patients with ACS and high risk of developing $\mathrm{CIN}$. The objective of this study is to determine whether NGAL measured 6 hours post PCI can identify patients that will develop CIN and require hemodialysis. Moreover, we will assess whether NGAL levels are associated with a longer length of hospital stay and hospital mortality.

\section{Methods}

A prospective observational study of consecutively admitted patients with ACS that underwent $\mathrm{PCl}$ was performed during two consecutive years at Shaio Clinic, a high-volume cardiovascular referral center in Bogota, Colombia. The local institutional ethics committee approved the study. Informed consent was obtained from all study subjects prior to enrollment. Not study procedures were performed before patient enrollment, and official inform consent was obtained.

$\mathrm{PCl}$ was performed using a standard protocol via either the radial or femoral artery approach by an attending interventional cardiologist. All procedures were performed using a standard dose $(3-5 \mathrm{ml} / \mathrm{kg})$ of non-ionic, low-osmolality contrast media in doses adjusted for body weight and type of cardiovascular angiogram. Patients' interventions were performed according to local and international guidelines, not per protocol. It is essential to highlight that all patients were monitored during PCI by a certified anesthesiologist to prevent cardiovascular complications; none of the subjects included in the study developed hypotension nor cardiovascular instability during the procedure. The decision to begin 
hemodialysis therapy was determined by the nephrology team taking into account the following lifethreatening indications for renal replacement therapy: acidosis unresponsive to medical treatment, acute severe refractory hyperkalemia, pulmonary edema and uremic complications (e.g., encephalopathy and uremic pericarditis) [31]. The nephrology team did not have access to the NGAL values during the study.

\subsection{Subjects}

The study inclusion criteria were age $>18$ years, ACS per standard definition [32], and high risk for CIN determined by a Mehran score $>11$ points (Table 1 ).

Exclusion criteria were end-stage renal disease requiring chronic hemodialysis (HD) or continuous ambulatory peritoneal dialysis (CAPD); suspected infection, sepsis or septic shock; exposure to nephrotoxic drugs or intravenous contrast medium 48 hours prior to the study period; terminal disease (malignant cancer of any type or end-stage liver disease); and pregnancy.

\subsection{Definition of contrast-induced nephropathy (CIN)}

CIN was defined according to criteria by the Acute Kidney Injury Network (AKIN) as an increase in SCr by $\geq 0.3 \mathrm{mg} / \mathrm{dl}$ ( $\geq 26.4 \mu \mathrm{mol} / \mathrm{L}$ ) or $\geq 1.5$ times baseline creatinine level within 48 hours of the procedure [33]. Urine volume criteria for AKI were not applied in this study because of potential changes in urinary volume induced by diuretics in the ICU. The estimated glomerular filtration rate (eGFR) was calculated using the Modification of Diet in Renal Disease-4 (MDRD) formula [34].

\subsection{Enrollment and Follow-Up}

After screening all patients for eligibility at the time of ICU admission, patients with high risk for CIN were treated prophylactically according to the institutional protocol for renal protection: normal saline at a rate of $1 \mathrm{~mL} / \mathrm{Kg} / \mathrm{h} \mathrm{IV}$; N-acetylcysteine 1,200 mg IV BID on the day before and 12 hours after the procedure, and sodium bicarbonate started at least 1 hour before the procedure and up to 6 hours after administration of contrast. All patients were followed daily until hospital discharge, and data were gathered daily using an electronic case report form for each patient.

\subsection{Clinical Outcomes}

Our primary aim was to determine the association of serum NGAL levels 6 hours after contrast media exposure and the need for hemodialysis after PCI in patients with ACS. Our secondary outcome was to determine the association of serum NGAL levels and hospital length of stay (LOS) or hospital mortality.

\subsection{Biomarker assay}

Venous blood samples measured serum creatinine ( $\mathrm{SCr}$ ) and blood urea nitrogen (BUN) every 24 hours after ICU admission. Blood was drawn prior to $\mathrm{PCl}$ and 6,24 , and 48 hours after the procedure to determine levels of SCr, BUN, and NGAL. Serum NGAL was measured using a commercially available kit 
(Alere $^{\mathrm{TM}}$ Triage ${ }^{\circledR}$ NGAL immunoassay), immediately after blood sample collection according to the manufacturer's instructions.

\subsection{Statistical Analysis}

We used Fisher's exact test to compare categorical variables and the non-parametric test (Man-Whitney $U$ Test) to evaluate continuous variables. Values are expressed as medians (IQR). Statistical significance was defined as a $p$-value $\leq 0.05$. A receiver operating characteristic (ROC) curve was developed to assess the accuracy of NGAL levels to -index to identify the best cut-off at 6 hours post PCl of NGAL. All statistical analyses were performed with IBM SPSS, Statistics for Mac, version 22.0. Armonk, NY: IBM Corp.

\section{Results}

A total of 2,875 PCls were performed during the study period. We identified 617 patients as potential study subjects; however, only 45 patients met the inclusion/exclusion criteria and were enrolled as study subjects (Figure 1). A majority of the subjects were men (60\%) and older than 70 years old. During the median 12.2 days of follow-up of all study subjects, 8 patients required hemodialysis therapy, and 3 patients died. None of the patients develop cardiogenic shock nor required cardiac surgery. The baseline characteristics of study subjects are presented in Table 2, and the serial measurements of NGAL, creatinine, and BUN at baseline, 6, 24, and 48 hours are shown in Figure 2.

Among the patients that required versus did not require hemodialysis, there were no differences in hemoglobin levels or platelets counts. However, subjects in the hemodialysis group had higher albumin levels, lower glomerular filtration rates at admission determined per MDRD-4 formula, and lower left ventricular ejection fraction. These subjects also had a longer hospital LOS, and one subject continued to requiring hemodialysis at hospital discharge. Finally, among subjects that required hemodialysis, 3 died. Serum concentrations of NGAL at 6 hours were higher in patients whom died during hospital admission (341.0 mg/mL [311-350] vs. $171.5 \mathrm{mg} / \mathrm{mL}$ [100-230], $p=0.007$ ). However, serum concentrations of NGAL were not different in patients with CIN who died (340.0 mg/ml [220-392] vs. $341.0 \mathrm{mg} / \mathrm{mL}$ [311-341], $p=0.1$ ). As expected, patients that required hemodialysis had a longer hospital LOS (17 days [+/-5] vs. 10 days [+/-7], $p=0.008)$.

Regarding serum biomarker levels at 6 hours, the median (IQR) for serum concentration of NGAL was significantly higher in subjects that required hemodialysis versus those that did not require hemodialysis (340.5 mg/mL [235-384] vs. $169 \mathrm{mg} / \mathrm{mL}$ [100-210], $p=0.001$ ) (Figure 3). BUN was also higher in the hemodialysis group versus those subjects not requiring hemodialysis, but the difference was not statistically significant. The median serum concentration of creatinine at 6 hours was similar between subjects that required hemodialysis versus those that did not require hemodialysis (1.55 [1.22-2.07] vs. 1.40 [1.20-1.70], $p=0.37)$. These data demonstrated that elevated serum levels of NGAL at 6 hours predicted the need for hemodialysis with an area under the curve (AUC) of 0.858 . Using the Youden index, 
we identified that the best cut-off at 6 hours post PCl of NGAL is $281 \mathrm{mg} / \mathrm{dL}$. With this cut-off, the sensitivity and specificity were 0.75 and 0.95 , respectively, to predict the requirement for hemodialysis.

\section{Discussion}

To the best of our knowledge, this is the first study available in medical literature to assess the role of serum NGAL as an early biomarker of $\mathrm{CIN}$ after PCl in high-risk patients. The main finding of our study is that serum levels of creatinine and BUN, traditional biomarkers of renal injury, could not detect early development of CIN. In contrast, serum concentrations of NGAL at 6 hours post-PCI could detect patients that are at high risk of developing $\mathrm{CIN}$ requiring hemodialysis during hospitalization. Moreover, we also found that NGAL levels were higher in patients who developed CIN and died during hospital admission. This finding is clinically relevant because using serum NGAL concentration, physicians could identify patients at risk of developing $\mathrm{CIN}$ that will require hemodialysis early and may benefit from prompt renal specialist consultation and medical interventions, such as optimization of intravascular volume status and avoidance of nephrotoxic medications.

Although our study is the first to use serum NGAL for early detection of CIN in high-risk patients with ACS undergoing PCl; our findings build on prior knowledge that has shown that NGAL is superior to serum creatinine or BUN for early diagnosis of acute kidney injury and CIN. A recent meta-analysis showed that NGAL had an excellent predictive utility for CIN with AUCs of 0.91 for serum NGAL and 0.94 for urinary NGAL [35]. However, in this meta-analysis, authors only included 4 studies that used serum NGAL in adults, and all 4 studies had small sample sizes [35]. Additionally, these studies were in patients who underwent elective $\mathrm{PCl}$, and only one study used the same definition of $\mathrm{CIN}$ as our study. Besides this, regarding renal protection protocols, in two of them, patients received normal intravenous saline at a rate of $1 \mathrm{~mL} / \mathrm{kg}$ per hour previous to the procedure and in only one study patients also received oral $\mathrm{N}$ acetylcysteine (NAC) $600 \mathrm{mg}$ twice daily for 3 days [28, 30,36]. A more recent study by Nguyen et al. with unselected patients with ST-elevation myocardial infarction treated by $\mathrm{PCl}$ and were all patient receive only $1000 \mathrm{~mL}$ of physiological saline given at a rate of $0.6 \mathrm{~mL} / \mathrm{kg}$ per hour for 24 hours except in those patients in Killip class III or IV; plasmatic NGAL did not provide additional value regarding CIN prediction compared with other risk [37].

In contrast, all of our study subjects received per-protocol treatment for renal protection to prevent CIN that included normal saline at a rate of $1 \mathrm{ml} / \mathrm{Kg} / \mathrm{h}$ IV; N-acetylcysteine 1,200 mg IV BID 24 hours before and 12 hours after the procedure, and sodium bicarbonate started at least 1-hour pre-procedure and continued up to 6 hours post-procedure. Moreover, our study included only high-risk patients identified by Mehran score $>11$ points, whereas other studies included lower-risk patients, which constitutes the strength and novelty of our results.

The importance of early detection of CIN is that several observational studies have demonstrated that inhospital mortality is five times higher in patients with CIN that patients who do not have CIN. Added to the above, observational studies have shown that as many as $20 \%$ of patients who develop CIN suffer a 
persistent worsening of renal function $[5,38,39]$. Therefore, early detection of CIN could mean an early consultation to a renal specialist because it has been demonstrated that a delay in nephrology consultation contributes to higher mortality in acute kidney injury [40].

Several urinary and serum biomarkers have been proposed to identify patients at risk of dying due to CIN. However, the results are controversial and not conclusive. In our study, we found that patients with higher concentrations of serum NGAL had higher mortality. Even though this is an exciting finding, our study was not powered nor designed to predict mortality; thus, this finding should be interpreted with caution. However, this might open the possibility to design more prominent, multicentric, prospective studies to evaluate whether NGAL may be used as a prognosis biomarker in patients with CIN and, more specifically, in patients with AKI due to CIN.

Our study has important strengths and limitations that need to be recognized. One of the strengths of our study is that the setting was a highly specialized cardiovascular hospital with established protocols to identify and intervene on patients at risk of CIN. Another strength of our study is the inclusion of only high-risk patients, an important group of patients frequently excluded from other studies. However, it is essential to recognize that the small sample size limits our study due to the recruitment of a specific highrisk patient population. This also limits our ability to the performance of all statistical tests to assess the performance of NGAL as a diagnostic marker in the early detection of patients with CIN requiring hemodialysis. Moreover, only three patients died in our study; thus, we cannot conclude, nor hypothesize the role of NGAL in this regard. It is important to mention that NGAL has been recently associated with heart failure and coronary artery disease possible as a manifestation of inflammation [41]; and essential aspect that needs to be kept in mind because this condition could develop a false positive scenario. Besides this, some patients that required hemodialysis had higher baseline $\mathrm{s} C \mathrm{r}$, which could imply some undiagnosed cases of the chronic kidney, which could have an impact on the initial value of NGAL. Nevertheless, the discriminatory power of NGAL at 6 hours superior to creatinine levels at 6 hours to predict the need for hemodialysis. Acute kidney injury is a widely used and accepted definition. However, there are several definitions, and there is controversy about which is the most representative classification. Thus, we did not asses AKI in patients that did not require hemodialysis. Readers should be aware that the data presented in this study only represent patients with severe CIN requiring hemodialysis, and these data should not be extrapolated to other patients. Finally, not all hospitals can ensure nephroprotection in high-risk patients within the first 6 hours of hospital admission. Therefore, the results presented in this study might not be generalizable for all hospitals.

\section{Conclusion}

In summary, our study has demonstrated that in patients with ACS undergoing PCl that are at high risk of developing CIN, an elevated serum level of NGAL 6 hours after contrast exposure predicts the development of acute renal failure requiring hemodialysis. Early detection of acute kidney injury may prompt clinicians to seek early renal consultation and initiate aggressive therapies to reduce the risk of 
progression of renal failure. Additional studies are needed to confirm our findings and identify potential therapeutic interventions that may delay the progression of $\mathrm{CIN}$ in high-risk patients.

\section{Abbreviations}

Intensive care unit (ICU)

Neutrophil Gelatinase Associated Lipocalin (NGAL)

Contrast-induced nephropathy (CIN)

Acute coronary syndrome (ACS)

Percutaneous coronary intervention (PCl)

Kidney disease (CKD)

Diabetes mellitus (DM)

Congestive heart failure (CHF)

Serum creatinine (SCr)

Hemodialysis (HD)

Continuous ambulatory peritoneal dialysis (CAPD)

Estimated glomerular filtration rate (eGFR)

Modification of Diet in Renal Disease-4 (MDRD)

Length of stay (LOS)

\section{Declarations}

Conflict of interest: All authors have no conflict of interest

Ethics approval and consent to participate: This study was approved by the Committee of the Research Ethics of the Fundacion Clinica Shaio and Universidad de La Sabana. The purpose of the study was explained and written informed consent was obtained from each study participant or his or her caregivers. The patient's privacy and the availability of services were guaranteed by the investigator team even if the patients decided to leave the study. This study did not involve any potentially harmful intervention for the patient. The confidentiality of the data was preserved until the writing of the manuscript for publication. 
Consent for publication: Not applicable.

Competing interests: The authors declare that they have no competing interests.

Availability of data and materials: Not applicable

Funding: Not applicable

Author contribution: LFR, DFSB, CAB, SM, NJS, MP, EG, RB and AR contributed to the planning, writing and, correction of each section of the manuscript. All authors have read and approved the manuscript.

Acknowledgements: Special thanks to all patients who agreed to participate in the study

\section{References}

1. Rihal CS, Textor SC, Grill DE et al. Incidence and prognostic importance of acute renal failure after percutaneous coronary intervention. Circulation 105(19), 2259-2264 (2002).

2. Dangas G, Gdangas@Crf.Org, lakovou I et al. Contrast-Induced nephropathy after percutaneous coronary interventions in relation to chronic kidney disease and hemodynamic variables. American Journal of Cardiology 95(1), 13-19 (2005).

3. Parfrey PS, Griffiths SM, Barrett BJ et al. Contrast material-induced renal failure in patients with diabetes mellitus, renal insufficiency, or both. A prospective controlled study. N Engl J Med 320(3), 143-149 (1989).

4. Marenzi G, Lauri G, Assanelli E et al. Contrast-induced nephropathy in patients undergoing primary angioplasty for acute myocardial infarction. J Am Coll Cardiol 44(9), 1780-1785 (2004).

5. Mccullough PA, Wolyn R, Rocher LL, Levin RN, O'neill WW. Acute renal failure after coronary intervention: incidence, risk factors, and relationship to mortality. Am J Med 103(5), 368-375 (1997).

6. Ozkok S, Ozkok A. Contrast-induced acute kidney injury: A review of practical points. World J Nephrol 6(3), 86-99 (2017).

7. Nash K, Hafeez A, Hou S. Hospital-acquired renal insufficiency. Am J Kidney Dis 39(5), 930-936 (2002).

8. Murphy SW, Barrett BJ, Parfrey PS. Contrast nephropathy. J Am Soc Nephrol 11(1), 177-182 (2000).

9. Liu KD, Brakeman PR. Renal repair and recovery. Crit Care Med 36(4 Suppl), S187-192 (2008).

10. Coca SG, Yalavarthy R, Concato J, Parikh CR. Biomarkers for the diagnosis and risk stratification of acute kidney injury: a systematic review. Kidney Int 73(9), 1008-1016 (2008).

11. Siew ED, Ware LB, Ikizler TA. Biological markers of acute kidney injury. J Am Soc Nephro/ 22(5), 810820 (2011).

12. Mehran R, Aymong ED, Nikolsky E et al. A simple risk score for prediction of contrast-induced nephropathy after percutaneous coronary intervention: development and initial validation. J Am Coll Cardiol 44(7), 1393-1399 (2004). 
13. Bartholomew BA, Harjai KJ, Dukkipati S et al. Impact of nephropathy after percutaneous coronary intervention and a method for risk stratification. Am J Cardio/ 93(12), 1515-1519 (2004).

14. Harjai KJ, Raizada A, Shenoy $C$ et al. A comparison of contemporary definitions of contrast nephropathy in patients undergoing percutaneous coronary intervention and a proposal for a novel nephropathy grading system. Am J Cardiol 101(6), 812-819 (2008).

15. Brown JR, Devries JT, Piper WD et al. Serious renal dysfunction after percutaneous coronary interventions can be predicted. Am Heart J 155(2), 260-266 (2008).

16. Fu N, Li X, Yang S et al. Risk score for the prediction of contrast-induced nephropathy in elderly patients undergoing percutaneous coronary intervention. Angiology 64(3), 188-194 (2013).

17. Sgura FA, Bertelli L, Monopoli D et al. Mehran contrast-induced nephropathy risk score predicts shortand long-term clinical outcomes in patients with ST-elevation-myocardial infarction. Circ Cardiovasc Interv 3(5), 491-498 (2010).

18. Wi J, Ko YG, Kim JS et al. Impact of contrast-induced acute kidney injury with transient or persistent renal dysfunction on long-term outcomes of patients with acute myocardial infarction undergoing percutaneous coronary intervention. Heart 97(21), 1753-1757 (2011).

19. Wi J, Ko YG, Shin DH et al. Prediction of Contrast-Induced Nephropathy With Persistent Renal Dysfunction and Adverse Long-term Outcomes in Patients With Acute Myocardial Infarction Using the Mehran Risk Score. Clin Cardio/36(1), 46-53 (2013).

20. Aykan A, Gül I, Gökdeniz T et al. Is coronary artery disease complexity valuable in the prediction of contrast induced nephropathy besides Mehran risk score, in patients with ST elevation myocardial infarction treated with primary percutaneous coronary intervention? Heart Lung Circ 22(10), 836-843 (2013).

21. Kjeldsen L, Bainton DF, Sengeløv H, Borregaard N. Identification of neutrophil gelatinase-associated lipocalin as a novel matrix protein of specific granules in human neutrophils. Blood 83(3), 799-807 (1994).

22. Cowland JB, Borregaard N. Molecular characterization and pattern of tissue expression of the gene for neutrophil gelatinase-associated lipocalin from humans. Genomics 45(1), 17-23 (1997).

23. Mishra J, Dent $C$, Tarabishi R et al. Neutrophil gelatinase-associated lipocalin (NGAL) as a biomarker for acute renal injury after cardiac surgery. Lancet 365(9466), 1231-1238 (2005).

24. Mishra J, Ma Q, Prada A et al. Identification of neutrophil gelatinase-associated lipocalin as a novel early urinary biomarker for ischemic renal injury. J Am Soc Nephrol 14(10), 2534-2543 (2003).

25. Bachorzewska-Gajewska H, Malyszko J, Sitniewska E, Malyszko JS, Dobrzycki S. Neutrophilgelatinase-associated lipocalin and renal function after percutaneous coronary interventions. Am J Nephrol 26(3), 287-292 (2006).

26. Schmidt-Ott KM, Mori K, Li JY et al. Dual action of neutrophil gelatinase-associated lipocalin. J Am Soc Nephrol 18(2), 407-413 (2007).

27. Haase M, Bellomo R, Devarajan P, Schlattmann P, Haase-Fielitz A, Group NM-al. Accuracy of neutrophil gelatinase-associated lipocalin (NGAL) in diagnosis and prognosis in acute kidney injury: 
a systematic review and meta-analysis. Am J Kidney Dis 54(6), 1012-1024 (2009).

28. Liu XL, Wang ZJ, Yang Q et al. Plasma neutrophil-gelatinase-associated lipocalin and cystatin C could early diagnose contrast-induced acute kidney injury in patients with renal insufficiency undergoing an elective percutaneous coronary intervention. Chinese medical journal 125(6), 10511056 (2012).

29. Liebetrau C, Gaede L, Doerr O et al. Neutrophil gelatinase-associated lipocalin (NGAL) for the early detection of contrast-induced nephropathy after percutaneous coronary intervention. Scandinavian journal of clinical and laboratory investigation 74(2), 81-88 (2014).

30. Alharazy SM, Kong N, Saidin R et al. Neutrophil gelatinase-associated lipocalin as an early marker of contrast-induced nephropathy after coronary angiography. Angiology 65(3), 216-223 (2014).

31. Khwaja A. KDIGO clinical practice guidelines for acute kidney injury. Nephron Clin Pract 120(4), c179184 (2012).

32. Thygesen K, Alpert JS, Jaffe AS et al. Third universal definition of myocardial infarction. Circulation 126(16), 2020-2035 (2012).

33. Mehta RL, Kellum JA, Shah SV et al. Acute Kidney Injury Network: report of an initiative to improve outcomes in acute kidney injury. Crit Care11(2), R31 (2007).

34. Levey AS, Bosch JP, Lewis JB, Greene T, Rogers N, Roth D. A more accurate method to estimate glomerular filtration rate from serum creatinine: a new prediction equation. Modification of Diet in Renal Disease Study Group. Ann Intern Med 130(6), 461-470 (1999).

35. Wang K, Duan CY, Wu J et al. Predictive Value of Neutrophil Gelatinase-Associated Lipocalin for Contrast-Induced Acute Kidney Injury After Cardiac Catheterization: A Meta-analysis. Can J Cardiol 32(8), 1033.e1019-1029 (2016).

36. Padhy M, Kaushik S, Girish MP, Mohapatra S, Shah S, Koner BC. Serum neutrophil gelatinase associated lipocalin (NGAL) and cystatin $\mathrm{C}$ as early predictors of contrast-induced acute kidney injury in patients undergoing percutaneous coronary intervention. Clinica chimica acta; international journal of clinical chemistry 435 48-52 (2014).

37. Nguyen LS, Spagnoli V, Kerneis M et al. Evaluation of neutrophil gelatinase-associated lipocalin and cystatin $\mathrm{C}$ as biomarkers of acute kidney injury after ST-segment elevation myocardial infarction treated by percutaneous coronary intervention. Arch Cardiovasc Dis 112(3), 180-186 (2019).

38. Rear R, Bell RM, Hausenloy DJ. Contrast-induced nephropathy following angiography and cardiac interventions. doi:10.1136/heartjnl-2014-306962 (2016).

39. Maioli M, Toso A, Leoncini M, Gallopin M, Musilli N, Bellandi F. Persistent Renal Damage After Contrast-Induced Acute Kidney Injury. doi:10.1161/CIRCULATIONAHA.111.085290 (2012).

40. Soares DM, Pessanha JF, Sharma A, Brocca A, Ronco C. Delayed Nephrology Consultation and High Mortality on Acute Kidney Injury: A Meta-Analysis. Blood Purification 43(1-3), 57-67 (2017).

41. Lahiri A, Alex AG, George PV. Estimating the prevalence of elevated plasma neutrophil gelatinase associated lipocalin level in patients with acute coronary syndromes and its association with outcomes. Indian Heart J 70(2), 220-224 (2018). 


\section{Tables}

Table 1. The Mehran risk score for the prediction of CIN

Mehran score periprocedural CIN risk factor

Hypotension (SBP $<80 \mathrm{mmHg}$ or $<1$ hour of inotropic support)

Intra-arterial balloon pump therapy

Chronic heart failure (NYHA III/IV or recent pulmonary edema

Age $<75$ years

diabetes mellitus

Score

5

5

5

4

3

3

4

OR

Creatinine $>1.5 \mathrm{mg} / \mathrm{dL}$

Estimated glomerular filtration rate $<20 \mathrm{~mL} / \mathrm{min}$
Estimated glomerular filtration rate $20-40 \mathrm{~mL} / \mathrm{min}$

6

4

Estimated glomerular filtration rate $40-60 \mathrm{~mL} / \mathrm{min}$

Contrast media volume (cc)

2

1 point for each 100

CIN, contrast induce nephropathy; SBP, systolic blood pressure; NYHA, New York heart association functional classification; HCT, hematocrit.

*Only patients with a score $>11$ were included in the study

Table 2. Baseline characteristics of patients with acute coronary syndrome (ACS) stratified according to the requirement of hemodialysis during hospital admission 


\section{No Hemodialysis}

Hemodialysis

$p$

Characteristic

$(n=37)$

$19(51)$

Male

Age, median (IQR)

Comorbid conditions, $\mathbf{n}$ (\%)

Obesity

Hypertension

Active cancer

Atrial fibrillation

Chronic heart failure

COPD

Chronic kidney disease

Diabetes mellitus

Hyperlipidemia

Hypothyroidism

Tobacco use

At admission, median (IQR*)

Left ventricular ejection fraction

MDRD4

Creatinine

BUN

Hemoglobin

Platelets

Admission diagnosis, $\mathbf{n}$ (\%)

NSTEMI

STEMI

Unstable angina

Clinical outcomes, n (\%)

Discharge hemodialysis

In-hospital mortality $\begin{aligned} 24 & (65) \\ 3 & (8) \\ 10 & (27)\end{aligned}$

$0(0)$

$0(0)$

$$
\begin{gathered}
7(19) \\
30(81) \\
2(5) \\
4(10) \\
15(40) \\
4(10) \\
3(8) \\
16(43) \\
27(73) \\
10(27) \\
12(32)
\end{gathered}
$$

$45(21,55)$

$46(40,47)$

$1.15(1.2,1.6)$

$21(15,35)$

$14.10(12.6,15.9)$

$219(193,282)$
1 (12)

7 (87)

$0(0)$

2 (22)

$6(75)$

$0(0)$

1 (12)

5 (62)

6 (75)

3 (37)

$4(50)$

$32(30,53)$
$36(31,46)$

$1.75(1.2,2.1)$

$37(19,57)$

$12.55(11.5,14.1)$

$261(200,281)$
Value

0.01

0.41

0.66

0.66

0.31

0.28

0.07

0.37

0.30

0.32

0.90

0.55

0.34

0.89

0.14

0.27

0.09

0.09

0.63
7 (87)

1 (12)

0 (0)

0.21

0.69

0.95

1 (12)

3 (37)
0.03

$<0.01$

NSTEMI, non-ST-elevation myocardial infarction; STEMI, ST-segment elevation myocardial infarction IQR, Interquartile ratio; COPD chronic obstructive lung disease; BUN, Blood urea nitrogen; MDRD4, 4-variable Modification of Diet in Renal Disease Study Group formula

\section{Figures}




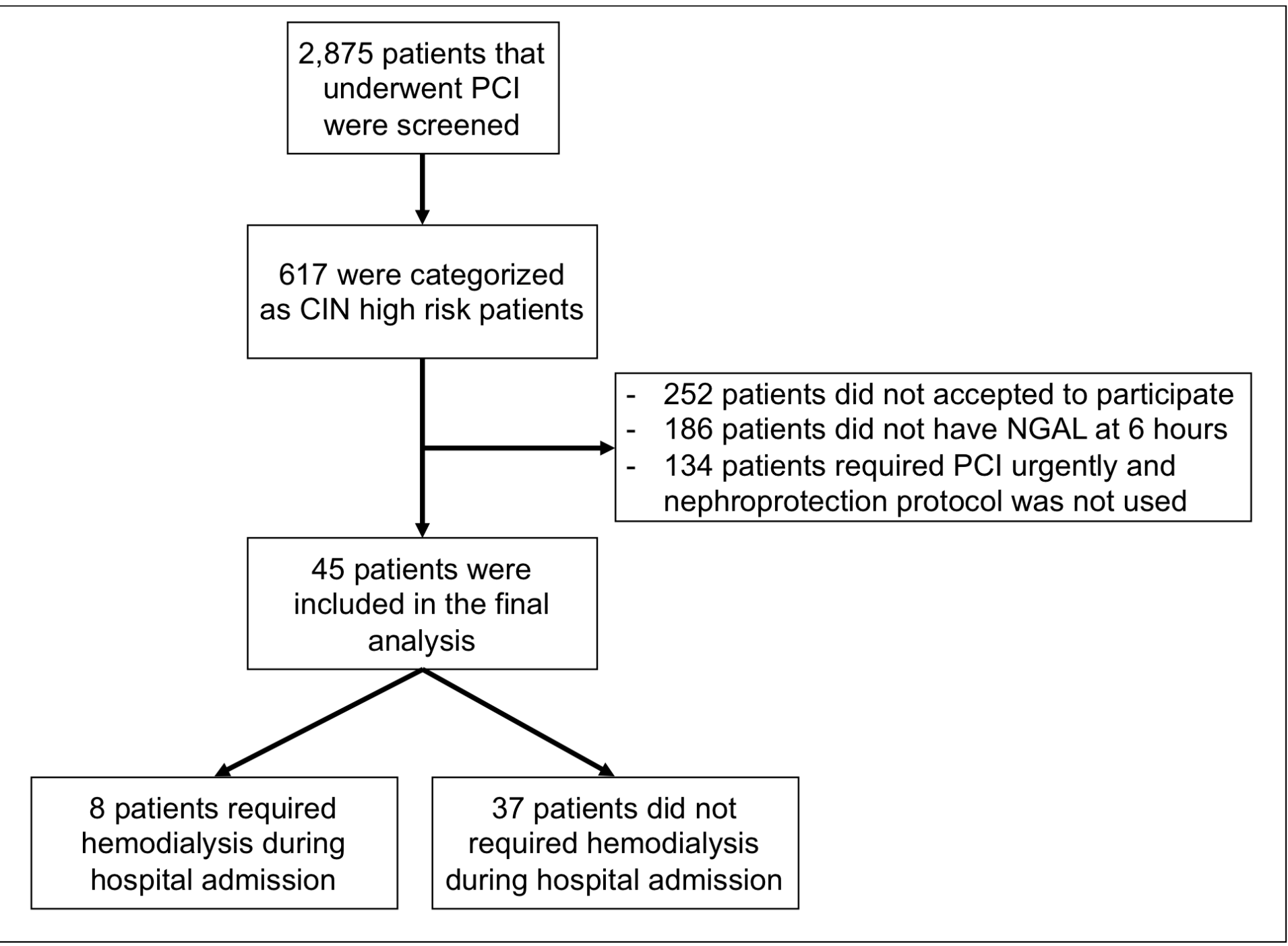

Figure 1

Study flow chart. Flow diagram of the patients with the acute coronary syndrome (ACS) that underwent PCl that entered the study.
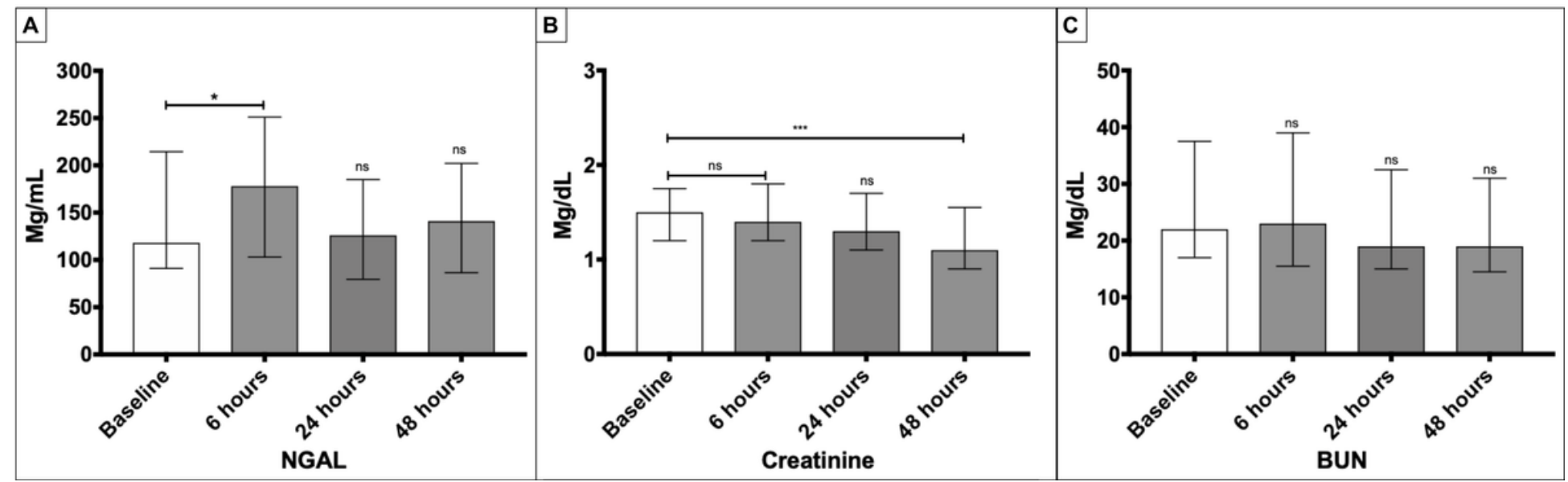

Figure 2 
Serial measurements of NGAL, creatinine, and BUN at baseline, 6,24 , and 48 hours. Box plots of serum NGAL, creatinine, and BUN at baseline, 6 hours, 24 hours and 48 hours. A. NGAL levels started to rise at 6 hours and then decreased at 24 and 48 hours. B. Creatinine values did not rise at any interval. C. BUN levels also did not rise at any time interval. NS: Not significant; P: $<0.05$.

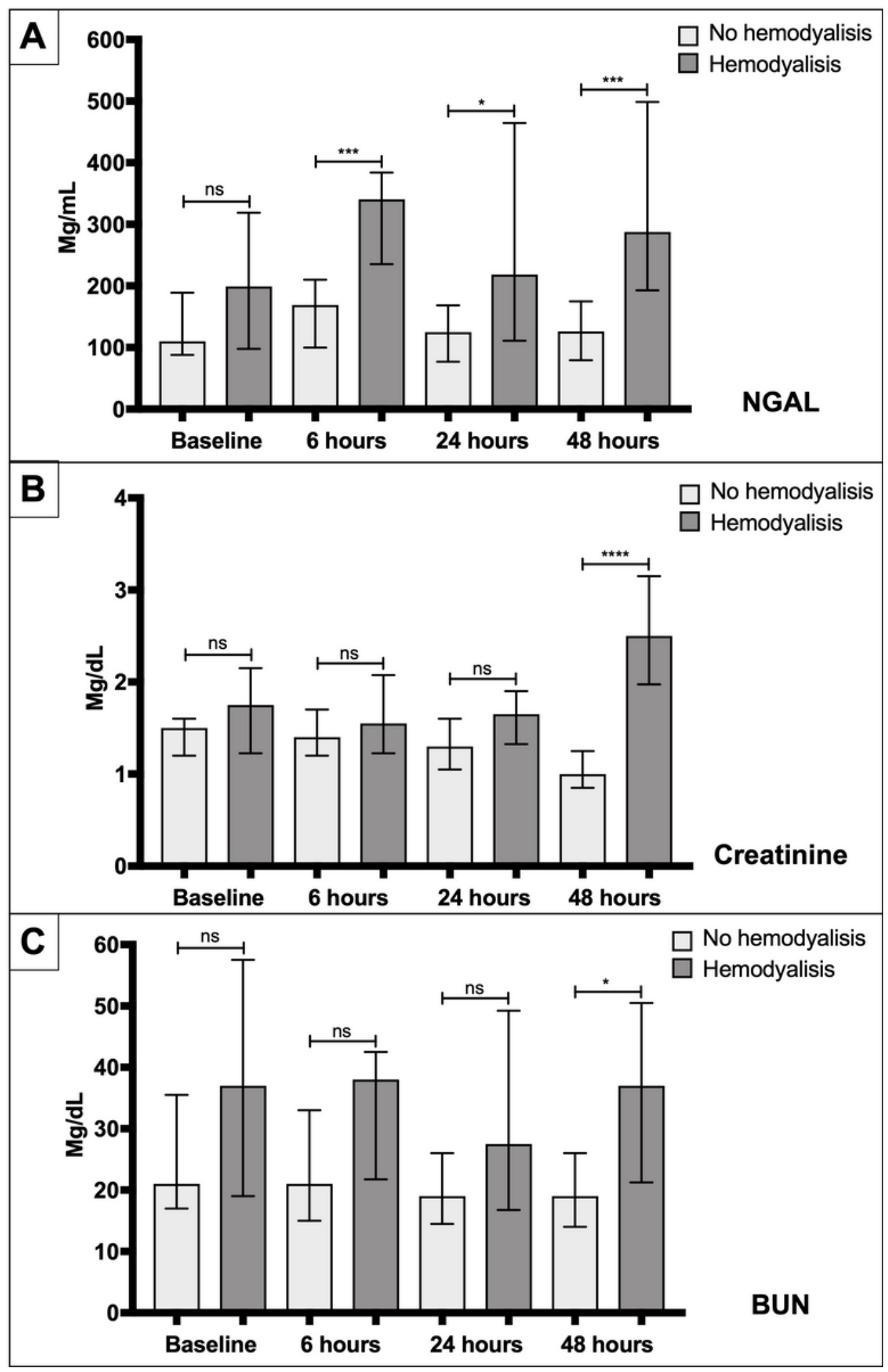

Figure 3 
Serial measurements of NGAL, creatinine, and BUN at baseline, 6,24 , and 48 hours in hemodialysis patients versus those who did not require hemodialysis. Box plots of serum NGAL, creatinine and BUN at baseline, 6 hours, 24 hours, and 48 hours in the patients who required hemodialysis and those who did not. A. NGAL levels started to rise at 6 hours with higher values in those patients who require hemodialysis. B. Creatinine values only started to rise at 48 hours in those patients who require hemodialysis. C. BUN levels did not show a rising pattern in either group. NS: Not significant; $\mathrm{p}:<0.05$. 